\title{
Predator and prey perception in copepods due to hydromechanical signals
}

\author{
Thomas Kiørboe*, André W. Visser \\ Danish Institute for Fisheries Research, Charlottenlund Castle, DK-2920 Charlottenlund, Denmark
}

\begin{abstract}
Copepods can perceive moving predators and prey by means of the hydrodynamical disturbances these generate. We formulate a simplified, general model of the fluid disturbance generated by a plankter that is moving or generating a feeding current and we estimate the magnitude and attenuation of the different components of the fluid disturbance. We use this model to argue that prey perception depends on the absolute magnitude of the fluid velocity generated by the moving prey, while predator perception depends on the magnitude of one or several of the components of the fluid velocity gradients (deformation rate, vorticity, acceleration) generated by the predator. On the assumption that hydrodynamic disturbances are perceived through the mechanical bending of sensory setae, we estimate the magnitude of the signal strength due to each of the fluid disturbance components. We then derive equations for reaction distances as a function of threshold signal strength and the size and velocity of the prey or predator. We provide a conceptual framework for quantifying threshold signal strengths and, hence, perception distances. The model is illustrated by several examples, and we demonstrate, for example, (1) how larval fish behaviour is adapted to allow their undetected approach up to the strike distance of their copepod prey, (2) that prey velocity is much more significant for prey encounter rates than traditionally assumed, even for cruising predators, (3) that prey perception is strongly biased towards large and rapidly swimming/sinking prey particles, and (4) that the model can accommodate the 3 orders of magnitude variation in clearance rates observed in the copepod Oithona similis feeding on motile protists and sinking particles. We finally discuss the implications of hydromechanical predator and prey perception to trophic interactions and vertical particle fluxes, and suggest important research questions that may be addressed.
\end{abstract}

KEY WORDS: Hydrodynamic model · Fish larval behaviour · Oithona feeding · Vertical flux

\section{INTRODUCTION}

Predator and prey perception are fundamental for trophic interactions in marine plankton, and prey or predator perception distances are critical to capture success and escape probability, respectively. Predator and prey perception in marine planktonic animals can be due to visual, mechanical or chemical signals. This study examines aspects of mechanical predator and prey perception with special emphasis on perception distances in copepods.

A body moving through water generates velocity gradients, the magnitude of which depends on the size and velocity of the moving body. This is the case whether the body moves due to a body force (gravity or

•E-mail: tk@dfu.min.dk buoyancy, e.g. a sinking faecal pellet or an ascending Noctiluca scintillans) or it is self propelled (e.g. a cruising copepod with a feeding current). A predator may perceive the fluid velocity generated by a moving prey (and hence attack), and a prey may perceive the velocity gradients generated by a moving predator (and hence escape). It has been demonstrated experimentally that copepods are able to perceive such fluid disturbances and to respond accordingly (Schröder 1967. Haury et al. 1980, Fields \& Yen 1996, 1997, Heuch \& Karlsen 1997), although it has not always been clear to which component of the fluid disturbance (fluid velocity, fluid acceleration, velocity gradient, or other characteristics) the copepod responds.

In this study, using idealised models, we attempt to quantify the magnitude and attenuation of the different components of the fluid disturbance generated by a moving body and to identify the component(s) that 
may be detected by a copepod. If one knows the magnitude of the fluid disturbance required to elicit a response, it is possible to estimate the distance at which a copepod can perceive a predator or a prey as a function of the size and velocity of the predator or prey.

Following Tiselius \& Jonsson (1990) and Tiselius et al. (1997), we examined the fluid velocity and velocity gradients generated by a moving plankter by treating the swimming organism as a translating sphere at low Reynolds number (i.e. laminar flow). We recognise that this is a great simplification, both because few plankters are spherical and because the analysis ignores hydrodynamical signals generated by moving appendages, which may also be important (Kirk 1985). However, this simplification makes the problem amenable to quantitative analysis and our results more general. Zaret (1980) developed a much more detailed model of the fluid disturbance generated by a specific organism (the cladoceran Bosmina) and a discussion of more complex real situations can be found in Yen \& Strickler (1996). Despite its simplicity, our quantitative analysis may help to identify some important characteristics of predator and prey perception, may have direct application to some special situations, and may help identify possible new research avenues. In the following we first provide models of fluid disturbance; then discuss which component(s) of the fluid disturbance may elicit response in different situations; and finally provide examples to illustrate the theory and discuss some of its potential implications for trophic interactions in the pelagic realm.

\section{CHARACTERIZATION OF THE FLOW FIELD: DEFINITION OF TERMS}

In our reading of the biological literature, it has become apparent to us that there is a considerable confusion in terminology and quantification of the different components of fluid motion. Shear, velocity gradient and deformation rate are often used synonymously; terms such as 'stretching deformation' and 'shear deformation' are used without definition and may not mean the same to all, and vorticity and strain remain a mystery to many. We will therefore start with an explanation of the terms we use in the following (a more rigorous treatment is given in Appendix 1).

In a moving fluid, the motion of a fluid element can be split up into 3 parts: translation as a rigid body, rotation as a rigid body, and deformation (Pedley 1997; Fig. 1). These can be quantified, respectively, by the 'fluid velocity' (field), the 'vorticity' (solid body rotation), and the 'deformation rate' (or rate of strain). Both the rotational and the deformation components are due to velocity gradients and, quantitatively, the veloc-

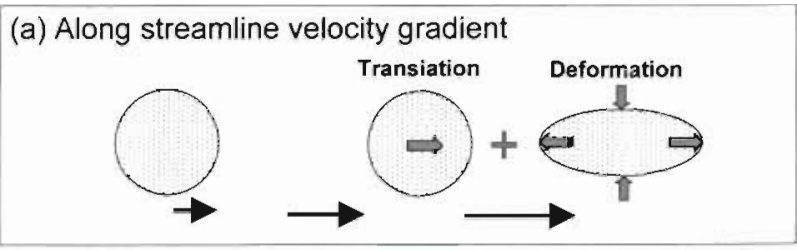

(b) Across streamline velocity gradient: Simple shear flow

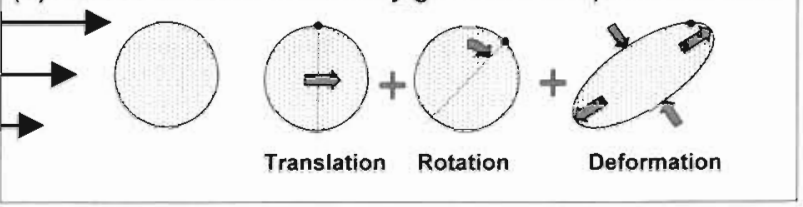

Fig. 1. Schematic of fluid motion. (a) An along streamline velocity gradient is characterised by translation and pure longitudinal deformation. (b) A simple shear flow is characterised by translation, rotation and pure shear deformation

ity gradient at any point in the fluid equals the sum of the deformation rate and the rotation.

Vorticity describes the rotational component of the flow. It has units of (radians) per unit time and can be viewed as solid body rotation. By definition, vorticity equals twice the rotation rate. Vorticity is a vector quantity and points 'upwards' from the plane where the rotation is anti-clockwise. In 3-D space, vorticity has 3 components. We shall call the axis about which there is maximum rotation, the principal axis of vorticity; vorticity around axes perpendicular to the principal axis is then zero. Vorticity is non-zero wherever there are velocity gradients perpendicular to the direction of the flow. Therefore, in a flow field where the velocity changes only in the direction of the flow, vorticity is zero (Fig. 1a). In a simple shear flow, i.e. where the velocity changes only in the direction perpendicular to the flow, the vorticity is equal to the velocity gradient (Fig. 1b).

Deformation can be visualised by considering a fluid-filled spherical balloon in a flow field. In a uniform flow with no velocity gradients, the balloon will keep its shape and the deformation rate is zero. In the presence of velocity gradients, different parts of the balloon will travel with different velocities, and the balloon will change shape; it deforms. However, the volume of the balloon remains constant because the fluid is incompressible. Therefore, if the balloon stretches in one direction, it will compress in others. To quantify deformation rate we need to define reference axes. Deformation rate, like vorticity, has units of inverse time. It is the velocity per unit distance at which the 2 points at the periphery of the balloon that are intersected by a particular reference axis move towards or away from one another. We shall define the 'maximum deformation rate' as the deformation rate 
along the axis that yields the fastest rate. The nature of deformation is independent of the direction of the velocity gradients; however, we may distinguish between pure 'shear deformation' and pure 'longitudinal deformation' which occur in flows characterised solely by simple shear or along-flow velocity gradients, respectively (Fig. 1).

The term 'shear' is often used in the biological literature, but it is only well defined in a simple shear flow (i.e. velocity gradients solely perpendicular to the flow direction), where it equals the magnitude of the velocity gradient. We shall refrain from using the term except in this special case.

In the following we provide equations for fluid velocity, deformation rate, vorticity and acceleration around a translating sphere, often without proof. We refer to a formal mathematical treatment in Appendix 1.

\section{MODEL OF THE FLOW FIELD}

Classic solutions of the low Reynolds number flow around a moving sphere (Stokes 1851, cf. Batchelor 1967) are often presented in terms of streamlines, either as a creeping flow around a fixed sphere (Fig. 2a), or for a translating sphere (Fig. 2b). Despite the different patterns of the stream lines, these 2 cases are in fact identical and are related by a coordinate transformation, i.e. coordinates fixed on the sphere (Fig. 2a) versus coordinates fixed with respect to the far field fluid (Fig. 2b). In this latter case, the streamlines are time dependent, i.e. some time later, the sphere together with the streamlines will have moved. How ever, Fig. 2 b can also be interpreted as the 'spherical pump' solution. That is, fluid is pumped at a uniform rate through a fixed spherical region of space. Therefore, the 2 different solutions in Fig. 2 allow 2 different interpretations in our context, viz.: (1) flow around a

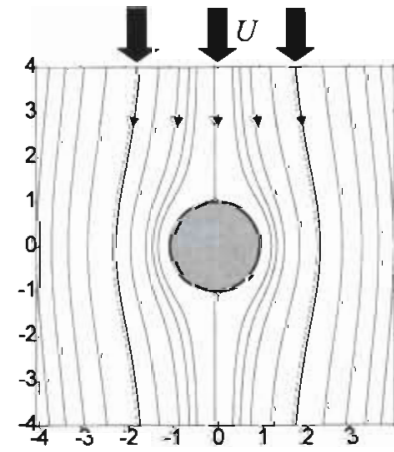

(a)

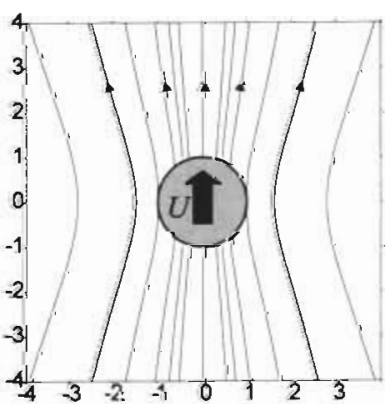

(b)
Fig. 2. Streamlines for creeping flow (a) around a fixed sphere or (b) around a translating sphere. Spatial coordinates are in units of sphere radii moving plankter (e.g. sinking or ascending particle, a swimming flagellate, the nose of an approaching fish); and (2) the flow field of a copepod feeding current.

The assumption of Stokes' flow (Fig. 2) is only strictly valid for Reynolds number $R e<1$, but yields accurate descriptions up to $R e \approx 1$ (White 1974). At higher Re the flow begins deviating significantly behind the particle. However, even up to quite high Reynolds numbers, of the order of 10 or more, there is limited effect upstream of the particle (cf. Lamb 1932, Batchelor 1967 for Oseen's correction, and Van Dyke 1988 for mapped flow fields up to Re order 10000). Because in this work we consider only the flow characteristics upstream of the equator of the particle, our considerations are robust up to at least $R e$ of the order of 10 . This is why the Stokes' flow (Fig. 2a) accurately represents the observed flow off the equator of an ascending Noctiluca scintillans ( $R e \approx 0.1$, Kiørboe \& Titelman 1998) or a descending marine snow particle $(R e \approx 2$, Ploug \& Jørgensen 1999). Likewise, and despite the simplicity of the model, the upstream flow characteristics of a copepod feeding current is well represented by the spherical pump solution, Fig. 2b, in Centropages typicus $(R e \approx 3$, Kiørboe et al. 1999 , in this issue), Euchaete rimana $(R e \approx 8$, Fields \& Yen 1997), the huge Pleuromamma xiphias ( $R e \approx 75$. Fields \& Yen 1993) and other copepods (Tiselius \& Jonsson 1990).

In the following we will concentrate on case (a) and note that most of the flow field characteristics for case (a) and (b) are identical; the only significant difference is the magnitude and distribution of acceleration. Table 1 explains the symbols used.

The stream function for creeping flow of velocity $U$ around a rigid sphere of radius $C$ is given in Appendix 1 (Eq. A15). The fluid velocity components in the radial and tangential directions are (cf. Eqs. A12 \& A13)

$$
\begin{aligned}
& u_{r}=-U \cos \theta\left(1-\frac{3 c}{2 r}+\frac{c^{3}}{2 r^{3}}\right) \\
& u_{\theta}=U \sin \theta\left(1-\frac{3 c}{4 r}-\frac{c^{3}}{4 r^{3}}\right)
\end{aligned}
$$

where $(r, \theta)$ are spherical coordinates (cf. Appendix 1). We have plotted the magnitude of the velocity, $|\mathbf{u}|=$ $\sqrt{ }\left(u_{r}^{2}+u_{\theta}^{2}\right)$, in Fig. $3 a$ and the attenuation of the velocity in the directions directly in front $\left(\theta=0^{\circ}\right)$ and off the equator $\left(\theta=90^{\circ}\right)$ of the sphere in Fig. 4a. High fluid velocities extend far into the fluid; even 15 radii in front of the sphere, the fluid velocity is still ca $10 \%$ of the sphere's velocity (or feeding current).

A general formulation of the maximum deformation rate, $\Delta(\theta, r)$, is given in Appendix 1 (Eqs. A4 to A8) and is plotted in Fig. 3b. Specific evaluations (from Eq. A.15) directly in front of the sphere (at $\theta=0^{\circ}$ ) and off its equator $\left(\theta=90^{\circ}\right)$ are 
Table 1. Glossary

\begin{tabular}{|c|c|}
\hline a & General acceleration \\
\hline$a_{\mathrm{r}}$ & Acceleration in the radial direction \\
\hline$a_{\theta}$ & Acceleration in the tangential direction \\
\hline$a^{*}$ & Acceleration threshold for response \\
\hline$\beta$ & Clearance rate \\
\hline$c$ & Radius of sphere; length scale of predator \\
\hline $\mathbf{e}$ & Rate of strain tensor \\
\hline$g$ & Gravitational acceleration \\
\hline$L$ & Length scale of perceiving organism ( radius) \\
\hline 1 & Offset of centre of mass and geometric centre of a body \\
\hline$r$ & Spherical coordinate; radial distance \\
\hline$R$ & Reaction distance \\
\hline$R_{\text {det }}, R_{\text {Shp }}, R_{\omega}, R_{y}$ & Reaction distance due to deformation, acceleration, vorticity, and shear, respectively \\
\hline$S$ & Signal strength; velocity difference \\
\hline$S^{*}$ & Threshold signal strength; velocity difference \\
\hline$S_{\text {det }}, S_{\text {slip }}, S_{\omega,} S_{y}$ & Signal strength due to deformation, acceleration, vorticity and shear, respectively; velocity difference \\
\hline$U$ & Velocity of sphere relative to far field fluid; velocity scale \\
\hline $\mathbf{u}$ & General fluid velocity \\
\hline$u_{\mathrm{r}}$ & Radial velocity \\
\hline$u_{\theta}$ & Tangential velocity \\
\hline$u^{*}$ & Threshold fluid velocity for response \\
\hline $\mathrm{v}$ & General particle velocity \\
\hline$\Delta$ & Maximum rate of deformation \\
\hline$\Delta^{*}$ & Threshold deformation rate for response \\
\hline$\delta_{1,2,3}$ & Principal components of rate of strain \\
\hline$\phi$ & Spherical coordinate; azimuthal angle \\
\hline$\gamma$ & Shear rate; does not have a robust definition \\
\hline$\mu$ & Dynamic viscosity \\
\hline$\theta$ & Spherical coordinate; polar angle \\
\hline$\rho$ & Density \\
\hline$\rho_{f}$ & Density of fluid \\
\hline$\rho_{p}$ & Density of particle \\
\hline$\psi$ & General stream function \\
\hline$\omega$ & Vorticity \\
\hline$\omega_{0}$ & Azimuthal (spherical coordinates) component of vorticity \\
\hline$\omega_{\mathrm{h}}$ & Horizontal component of vorticity \\
\hline$\omega_{h} \cdot$ & Critical horizontal vorticity for which an eccentric body begins to tumble \\
\hline$\omega^{\circ}$ & Threshold vorticity for response \\
\hline$\xi$ & Steady state orientation, angle relative to vertical \\
\hline$\Omega$ & Rotation rate \\
\hline$\nabla$ & Divergence operator \\
\hline$\partial$ & Partial derivative operator \\
\hline
\end{tabular}

$$
\begin{gathered}
\Delta\left(\theta=0^{\circ}\right)=3 U \mathrm{C}\left(r^{2}-c^{2}\right) /\left(2 r^{4}\right) \\
\Delta\left(\theta=90^{\circ}\right)=3 U C^{3} /\left(4 r^{4}\right)
\end{gathered}
$$

and are plotted in Fig. 4b. Deformation rate attenuates rapidly along the side of the particle, but extends much further in front of it. In addition, it has a maximum value $(3 U / 8 C)$ at a distance $(r=\sqrt{2} C)$ in front of the sphere.

In spherical coordinates, the vorticity has only 1 component, given by (Eq. A9)

$$
\omega_{\phi}=U \frac{3 c}{2 r^{2}} \sin \theta
$$

In contrast to deformation, vorticity is zero directly in front of the particle, and highest off its equator (Figs. $3 c$
$\& 4 c)$. The axis of the vorticity is in this 2 -D representation perpendicular to the plane. Vorticity extends around the equator of the particle as a doughnut of solid body rotation.

Fluid acceleration, $\mathbf{a}(r, \theta)=\mathbf{u} \cdot \nabla \mathbf{u}$, is everywhere proportional to the magnitude of the velocity gradient and to the curvature of the streamlines. Of the flow parameters, this is the only one that is different for the creeping flow and the feeding current (Fig. 3d,e). General equations for each case are given in Appendix 1 (Eq. A16 \& A20). At $\theta=0^{\circ}$ and $90^{\circ}$ the equation for the creeping flow simplifies to:

$$
\begin{aligned}
& a_{r}\left(\theta=0^{\circ}\right)=3 U^{2} c(r-c)^{3}(r+c)(2 r+c) /\left(4 r^{7}\right) \\
& a_{r}\left(\theta=90^{\circ}\right)=-3 U^{2} C(r-c)^{2}(r+c)\left(c^{2}+c r+4 r^{2}\right) /\left(16 r^{7}\right)
\end{aligned}
$$



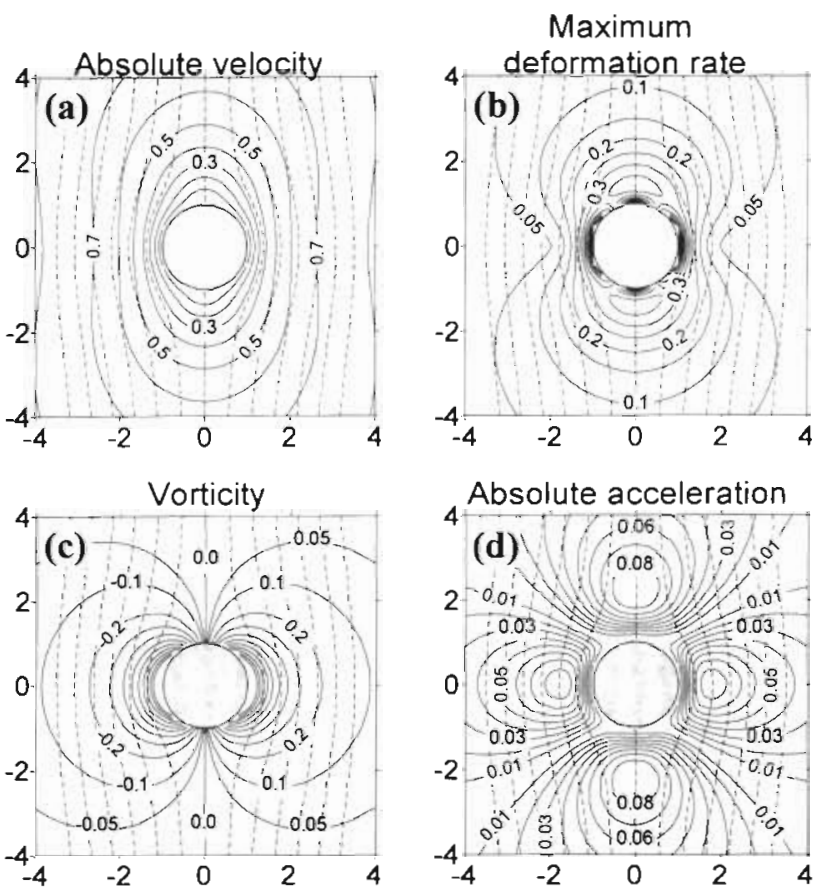

Absolute acceleration

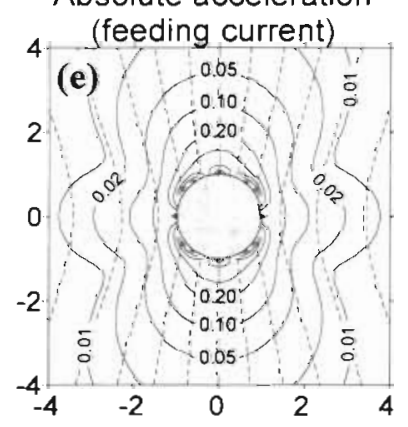

Fig. 3. Fluid flow around (a-d) a fixed sphere and (e) a translating sphere. Contour plots of (a) the absolute magnitude of the velocity measured relative to the particle, (b) maximum deformation rate, (c) vorticity and $(d, e)$ fluid acceleration. Spatial coordinates are in units of sphere radii $(c)$, velocity in units of body velocity $(U)$, deformation rate and vorticity in units of $U / C$ and acceleration in units of $U^{2} / c$

Acceleration for the creeping flow has lobes of high acceleration in front (at $r=2.2 \mathrm{c}$ ) and to the sides (at $r=1.77 \mathrm{C}$ ), with peak values of about $0.08 U^{2} / C$ (Figs. 3d \& 4d) The acceleration in both these locations is in the radial direction (the tangential components are both zero). However, the situations are somewhat different. At $\theta=0^{\circ}$, the acceleration is along the streamline so that fluid elements speed up and slow down. At $\theta=90^{\circ}$ acceleration is perpendicular to the streamlines and is manifest in the curvature of the fluid path.

\section{WHICH COMPONENTS OF THE FLUID DISTURBANCE CAN BE PERCEIVED?}

Copepods are equipped with hairs and setae extending in all directions, particularly on the antennules and the telson, and these function as mechanoreceptors (Strickler \& Bal 1973, Yen et al. 1992). In the following it is assumed that it is the mechanical 'bending' of setae extending into the fluid which is perceived by the copepod. Such bending may occur if there is a velocity difference between the copepod and the ambient fluid. The base of the setae moves with the copepod, whereas its tip moves with the local fluid velocity. The setae appear to be velocity rather than displacement sensors, and displacement rates of as little as $20 \mu \mathrm{m} \mathrm{s}^{-1}$ are enough to elicit a neurophysiological response in copepods (Yen et al. 1992). This does not necessarily imply that behavioural responses have the same threshold value. Sinking, swimming and other behaviours of a copepod may cause considerable velocity differences without eliciting escape responses. In the following we shall disregard velocity differences due to the organism's own behaviour, assuming that the copepod can distinguish these from fluid disturbances caused by a moving predator or prey.

In the following we shall distinguish between the perception of an approaching predator and a moving
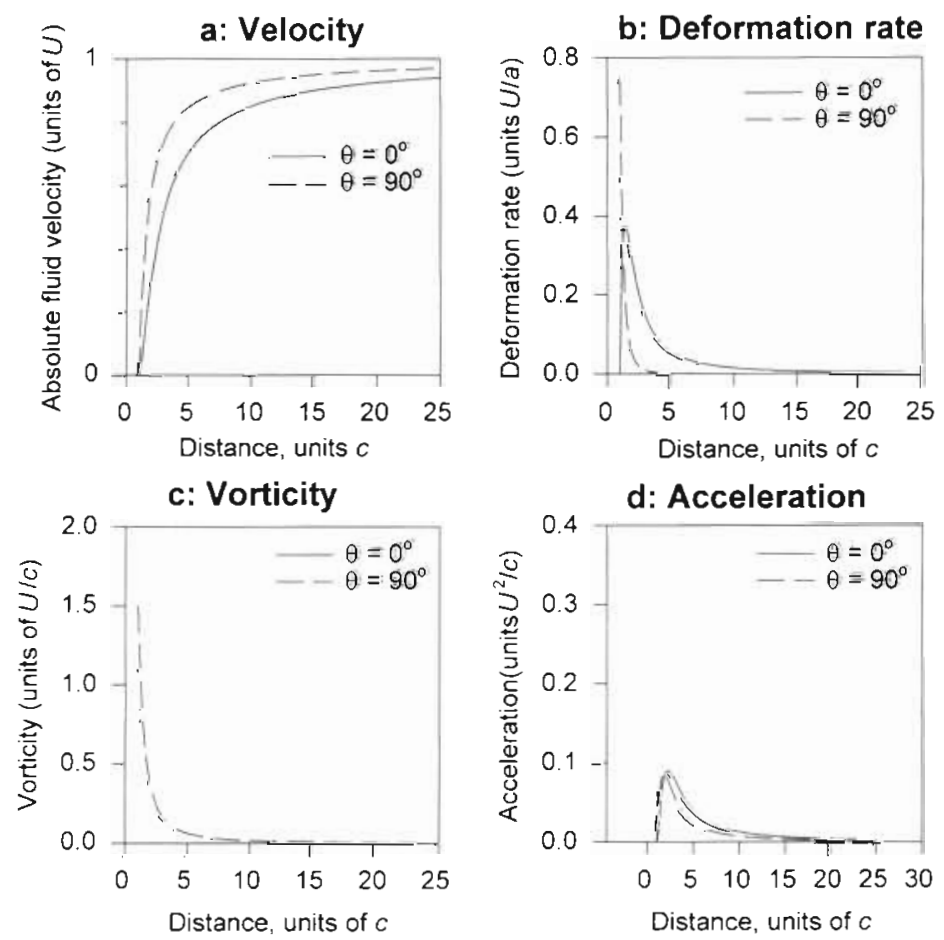

Fig. 4. Fluid velocity, deformation rate, vorticity and acceleration as functions of distance to a sphere (creeping flow solution); plotted in the move direction and perpendicular to the move direction off the equator (i.e. at $\theta=0$ and $90^{\circ}$ ) 
prey. Typically, a prey is much smaller than its predator. Thus, a prey will be more or less embedded in the flow generated by a predator; it will almost follow the streamlines. Conversely, the larger predator will not be moved by the local fluid disturbances generated by a small moving prey. Therefore, a large (predator) and a small (prey) particle, moving through the fluid, may be perceived by different components of the fluid disturbance.

\section{Prey perceiving predator}

Generally, all parts of a rigid prey particle almost following the streamlines of the flow in a velocity gradient cannot travel with the same velocity as the ambient fluid, and there will thus be a velocity difference between the prey and the fluid (Zaret 1980). A velocity difference can arise due to fluid deformation, vorticity and acceleration.

Deformation. The magnitude of the velocity difference (signal strength, $S$ ) due to deformation is (cf. Haury et al. 1980)

$$
S_{\text {def }}=L \Delta
$$

where $L$ is the linear dimension (radius) of the prey and $\Delta$ is the maximum deformation rate of the fluid. The rate of deformation also has components in other directions, but these will yield smaller signals and can, thus, be disregarded. Eq. (8) can also be interpreted as the differential between receptor signals on different parts of the body. This, in effect, results in Eq. (8) and more explicitly emphasises that multiple sensors are involved. The signal strength due to deformation is proportional to the deformation rate as well as the size of the prey. Its magnitude is independent of the direction of the velocity gradient, but may of course depend somewhat on the orientation of a (non-spherical) prey.

Vorticity. Vorticity may likewise cause a velocity difference depending on properties of the prey and on the orientation of the principal axis of vorticity (i.e. depending on the direction of movement of the predator). If the prey's centre of mass is identical to its geometric centre, then the prey will exactly follow the solid body fluid rotation, and there will be no velocity difference. However, most zooplankters have the centre of mass slightly offset from the geometrical centre (bottom-heavy), and they therefore resume a specific orientation in still water. If the steady state orientation is offset due to vorticity, the organisms will tend to reorientate due to the gravitational torque and assume a constant orientation where the gravitational torque is balanced by the shear induced viscous drag torque. The steady state orientation, measured as an angle rel- ative to the vertical, $\xi$, is (modified from Jonsson et al 1991)

$$
\sin \xi=\frac{3 \mu \omega_{h}}{1 \rho_{\mathrm{p}} g}
$$

where 1 is the distance between the centre of buoyancy and the centre of gravity, $\omega_{h}$ is the horizontal component of vorticity, $\mu$ the dynamic viscosity, $\rho_{p}$ the density of the prey, and $g$ the gravitational acceleration. If $\xi$ exceeds $90^{\circ}$, which happens if the viscous torque exceeds the gravitational torque for all orientations, there will be no steady state orientation, and the prey will continue to rotate with the fluid (but at a rate less that of the fluid). Since $\sin 90^{\circ}=1$, the critical vorticity for steady-state orientation is

$$
\omega_{\mathrm{h}}^{\cdot}=\frac{l \rho_{\mathrm{p}} g}{3 \mu}
$$

At vorticities less the critical value, the prey will assume its steady state orientation and, thus, will not rotate. The velocity difference due to vorticity $\left(S_{\omega}\right)$ can therefore be estimated as

$$
S_{\omega}=1 / 2 L \omega_{h}
$$

Recall that vorticity equals twice the rotation, hence the factor of $1 / 2$ in Eq. (11) (see also Eq. A1). At vorticities exceeding the critical value, the velocity difference due to vorticity does not exceed $\frac{1}{2} L \omega_{\mathrm{h}}$, irrespective of its actual magnitude. [If the predator is moving vertically, then $\omega_{\mathrm{h}}=\omega_{\phi}$; if the predator is moving horizontally, $\omega_{\mathrm{h}}=$ 0 laterally and $\omega_{\mathrm{h}}=\omega_{\phi}$ dorsally and ventrally].

Acceleration. In an accelerating fluid, a prey with a density different from that of the ambient water will travel at a different velocity. The magnitude of this 'slip velocity' can be estimated as follows. The simplified equation of motion (ignoring gravity) for a rigid sphere moving with velocity $\mathbf{v}$ in a fluid moving with velocity $\mathbf{u}$ is (Maxey \& Riley 1983)

$$
\frac{\mathrm{d} \mathbf{v}}{\mathrm{d} t}=\alpha(\mathbf{u}-\mathbf{v})+\beta \frac{\mathrm{d} \mathbf{u}}{\mathrm{d} t}
$$

where

$$
\alpha=\frac{9 \mu}{L^{2}\left(2 \rho_{p}+\rho_{\mathrm{f}}\right)}
$$

and

$$
\beta=\frac{3 \rho_{\mathrm{f}}}{2 \rho_{\mathrm{p}}+\rho_{\mathrm{f}}}
$$

where $\mu$ is the dynamic viscosity of the fluid and $\rho_{p}$ and $\rho_{\mathrm{f}}$ the density of the particle and the fluid, respectively.

If the fluid is under constant acceleration a (i.e. $\mathbf{u}=$ at), the steady state solution to Eq. (12) is similar to Stokes' law;

$$
S_{\text {slip }}=|\mathbf{a}|(\beta-1) / \alpha
$$

where $S_{\text {slip }}=|\mathbf{v}-\mathbf{u}|$ is the maximum slip velocity. It is not possible to solve Eq. (12) explicitly for a more realistic situation, when the acceleration instead of being 
constant is increasing over time as the prey is approached by the predator (cf. Figs. 3 \& 4). However, numerical solutions to Eq. (12) demonstrate that the actual slip velocity is consistently less than the above estimated $S_{\text {slip }}$ which thus provides an upper estimate of the slip velocity, also in a flow field with spatially varying acceleration. Because $\alpha$ varies with $L^{-2}$ (Eq. 13), signal strength due to this mechanism, $S_{\text {slip. }}$ scales with $L^{2}$.

Scaling. The 3 signal strengths attenuate differently as they are associated with different components of the flow (Figs. $3 \& 4$ ). The 'choice' of component therefore has implications on the distance at which a prey may perceive a predator. That is, different signals are perceived at different distances. We therefore compare the magnitudes of the different sources of velocity difference. For simplicity, we consider only the special case of $\theta=0^{\circ}$. Here, the vorticity is zero and the acceleration magnitude equals the velocity multiplied by the along-flow velocity gradient (which in this case $=\Delta$ ), i.e. $a_{r}=u_{r} \Delta$. Thus, from Eqs. (8) \& (15), the 2 sources of velocity difference, $S_{\text {def }}$ and $S_{\text {slip, }}$ are equal when

$$
L \Delta=u_{r} \cdot \Delta(\beta-1) / \alpha
$$

where $u_{r}$ is the critical fluid velocity magnitude at which the 2 sources of velocity difference are equal. Reorganising and combining with Eq. (13) yields

$$
u_{r}^{*}=\frac{L \cdot \alpha}{\beta-1}=\frac{9 \mu}{L\left(2 \rho_{\mathrm{p}}+\rho_{\mathrm{f}}\right)(\beta-1)} \propto L^{-1}
$$

Thus, the critical velocity is inversely proportional to the size of the prey organism. If one assumes that the copepod has an excess density of $5 \%, \rho_{i}=1 \mathrm{~g} \mathrm{~cm}^{-3}$ and $\mu=10^{-2} \mathrm{~g} \mathrm{~cm}^{-1} \mathrm{~s}^{-1}$, then $u_{r}^{*}=L^{-1} \mathrm{~cm}^{2} \mathrm{~s}^{-1}$ when $L$ is in $\mathrm{cm}$. For fluid velocities less the critical velocity, $S_{\text {der }}$ exceeds $S_{\text {shp }}$ and vice versa. For small prey, e.g. a $100 \mu \mathrm{m}$ nauplius, the critical fluid velocity $\left(-100 \mathrm{~cm} \mathrm{~s}^{-1}\right)$ exceeds that which can be generated by its predators ( $\leq$ swimming velocity), and $S_{\text {def }}$ provides the strongest signal. Conversely, for larger prey, e.g. a $1 \mathrm{~cm}$ copepod, one can imagine that its (correspondingly larger) predators may produce fluid velocities that exceed the critical velocity $\left(1 \mathrm{~cm} \mathrm{~s}^{-1}\right)$ and the slip velocity may, therefore, provide the strongest signal. Although true in a general sense, the latter situation is outside the model regime.

Although the magnitude of the velocity difference due to the different mechanisms may differ markedly, one cannot immediately rule out the weaker signal. The velocity difference due to slippage is homogeneous across the entire body of the prey while the velocity difference due to fluid deformation and vorticity varies across the body. Therefore, the copepod prey may be able to distinguish between the different types of signals. Whether a copepod responds to vorticity may depend on its normal behaviour. If it frequently reorientates (due to swimming and jumping) and subsequently rotates back towards steady state orientation, the velocity differences thus generated may be indistinguishable from signals due to predator induced vorticity. Clearly, observations and experiments are required to identify the relevant components and to estimate threshold values.

\section{Predator perceiving prey}

As noted above, the fluid disturbance generated by a small moving prey will not significantly move a large predator. Thus, the larger predator will not follow the streamlines of the flow generated by a small prey but rather be 'anchored' in the fluid (disregarding gravitational effects). Therefore, the fluid disturbance causes a velocity difference between any spot on the predator and the ambient fluid, which simply equals the (local) fluid velocity generated by the moving prey. We thus propose that the relevant characteristic of the fluid disturbance is the absolute magnitude of the fluid velocity rather than deformation rate or acceleration, i.e. signal strength $S_{u}=u$.

\section{PREY AND PREDATOR PERCEPTION DISTANCE}

In estimating perception distances, we will assume that the signal strength $(\sim$ velocity difference between fluid and animal) will have to exceed some threshold value, $S^{*}$, to elicit a response. This translates to threshold values for the different fluid components considered (from Eqs. 8, 11 \& 15), viz. $\Delta^{*}, \omega^{*}, a^{*}$, and $u^{*}$. By inserting these threshold values in the equations that describe the attenuation of the relevant characteristic of the fluid flow, one can estimate the perception distance $(R)$ by solving for $r$. Analytical solutions to the general equations given in Appendix 1 often do not exist, and therefore, below we only give solutions to the special cases of $\theta=0^{\circ}$ and $\theta=90^{\circ}$, i.e. directly in front of and directly off the equator of the moving plankter. Perception distances in other directions will be intermediate.

Deformation rate. At $\theta=0$, the reaction distance $R_{\text {def }}$ $=r\left(\Delta^{*}\right)$ due to deformation is found by solving Eq. (3)

$$
\begin{aligned}
& R_{\mathrm{def}}\left(\theta=0^{\circ}\right)=r\left(\Delta^{*}\right) \\
& =c \sqrt{\frac{3 U}{4 C \Delta^{*}}\left\{1+\sqrt{1-\frac{8 C \Delta^{*}}{3 U}}\right\}} \quad \text { for } \Delta^{*} \leq 3 U / 8 c
\end{aligned}
$$

(Eq. 3 actually has 2 solutions; Eq. 18 gives that furthest away from the sphere). Similarly at $\theta=90^{\circ}$ (cf. Eq. 4) 


$$
R_{\text {def }}\left(\theta=0^{\circ}\right)=\left(\frac{3 U c^{3}}{4 \Delta^{*}}\right)^{0.25}
$$

Vorticity. At $\theta=0^{\circ}$ vorticity is zero, while at $\theta=90^{\circ}$, the reaction distance due to vorticity is (cf. Eq. 5)

$$
R_{\omega}\left(\theta=0^{\circ}\right)=\left(\frac{3 U c}{2 \omega^{\circ}}\right)^{05}
$$

Vorticity and deformation. Assume that the copepod is able to perceive both vorticity and deformation: how should these 2 signals be considered simultaneously? At $\theta=90^{\circ}$, the velocity gradient is exactly perpendicular to the stream lines and thus equals the shear $(\gamma)$ :

$$
\gamma\left(\theta=0^{\circ}\right)=\frac{\partial u_{\theta}}{\partial r}=\frac{3 U c\left(I^{2}+c^{2}\right)}{4 r^{4}}=0.5 \omega+\Delta
$$

Signal strength in simple shear is

$$
\left.S_{\gamma}=\gamma L=(1 / 2 \omega)+\Delta\right) L
$$

and it thus follows that the signals from the 2 components are additive. The reaction distance at $\theta=90^{\circ}$ is found by solving Eq. 21 for $r$ at $\gamma=\gamma^{*}$ :

$$
R_{\gamma}\left(\theta=0^{\circ}\right)=\sqrt{\frac{\frac{3}{4} U C+\sqrt{\frac{9}{16} U^{2} C^{2}+3 \gamma^{\bullet} U C^{3}}}{2 \gamma^{\bullet}}}
$$

This somewhat special situation (simple shear flow, the copepod responding to both vorticity and deformation) is the only one in which shear may provide the triggering signal.
Fluid acceleration. We found no analytical solutions, but numerical solutions are given in Fig. 5.

Fluid velocity. The reaction distance equations above relate to aspects of the velocity gradients and are relevant to prey perceiving predators, while predators perceiving prey respond to the absolute fluid velocity magnitude. Fluid velocity relative to a stationary coordinate system, rather than relative to the moving sphere, is obtained by replacing the bracketed terms in Eqs. (1) \& (2) with 1 - the bracketed terms (Eqs. A18 \& A19). Solving these equations in our 2 directions for $r$ at $|u|=u^{*}$ yields

$$
R_{u}\left(\theta=0^{\circ}\right)=-c /\left(2 \cos \left(\left(4 \pi+\cos ^{-1}\left(u^{*} / U\right)\right) / 3\right)\right)
$$

and $\quad R_{u}\left(\theta=90^{\circ}\right)=c\left(-K^{-1 / 3}+K^{1 / 3}\right)^{-1}$

where $K=\left[2 u^{*} / U+\left(1+4\left(u^{*} / U\right)^{2}\right)^{0.5}\right]$

Fig. 5 illustrates the dependency of reaction distance on the velocity, size and threshold signal strength for the different flow characteristics. In all cases, $R$ is directly proportional to $c$, the size of the predator or prey, and increases monotonically with the translation or feeding current velocity. Deformation rate can be perceived at much longer distance in front of the predator than along its sides. However, if vorticity is perceived, the combined effect of vorticity and deformation rate is perceived about equally far in both directions.

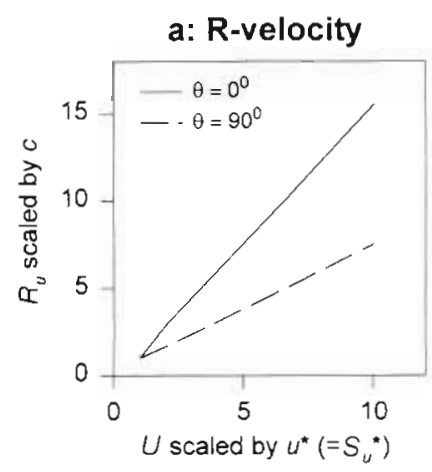

d: R-acceleration

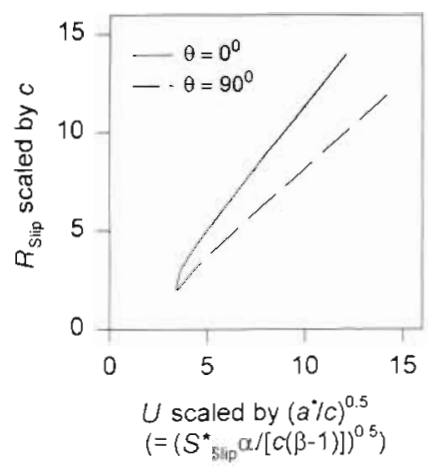

b: R-deformation
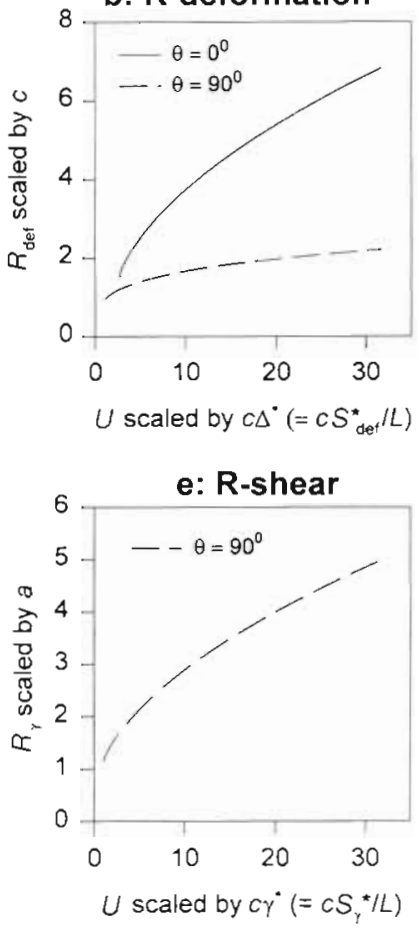

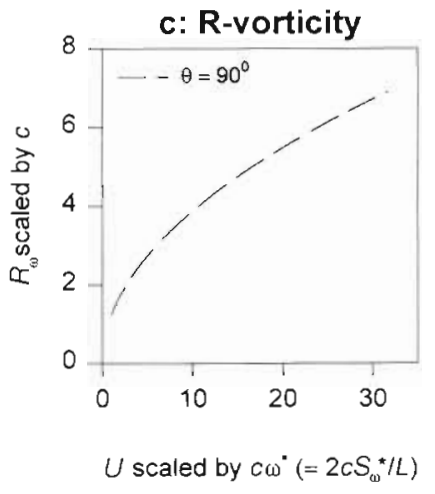

Fig. 5. Perception distance at $\theta=0$ and $90^{\circ}$ as a function of size ( $C$ and $L$ ), threshold signal strength $\left(S^{\circ}\right)$ and move velocity considering each of the components of the fluid disturbance 


\section{EXAMPLES AND ILLUSTRATION OF THE THEORY}

In the following we move from illustrations of details of the above model, to considerations of its more general implications

\section{Sources of velocity difference}

The observations of Yen \& Fields (1992) of the escape behaviour of copepod nauplii (Acartia hudsonica) entrained in the feeding current of a copepod may be used to illustrate the relative magnitude of the different sources of velocity difference between the prey and the ambient water. From their data we calculate an average velocity difference at the point of escape of $0.03 \mathrm{~cm} \mathrm{~s}^{-1}$. From the size of the nauplius $(250 \mu \mathrm{m})$, the estimated fluid acceleration at the point of escape $\left(0.05 \mathrm{~cm} \mathrm{~s}^{-2}\right)$, and by assuming a density difference of $5 \%$, we estimate a slip velocity $S_{\text {slip }} \approx 10^{-5} \mathrm{~cm} \mathrm{~s}^{-1}$ (Eq. 15). This is only a minute fraction of the velocity difference observed. The average fluid deformation rate at the point of escape can roughly be approximated by the root-mean-square of their estimated 'shear' and along stream line velocity gradient as 1.2 $( \pm 0.8) \mathrm{s}^{-1}$, which yields $S_{\mathrm{def}} \approx 0.015 \mathrm{~cm} \mathrm{~s}^{-1}$ (Eq. 8). This is of the same order as that observed, and the difference makes room for the effect of vorticity. The estimate is also similar to the threshold signal strength for deformation estimated for Acartia tonsa $\left(0.013 \mathrm{~cm} \mathrm{~s}^{-1}\right.$ ) (Kiørboe et al. 1999). Thus, for this small nauplius, fluid acceleration per se provides a much smaller signal than the fluid deformation rate, and the signal provided by the latter accords with that observed.

\section{Prey size effects on the capability of perceiving predator}

Independent of whether the characteristic of the flow to which a prey responds is fluid acceleration, fluid deformation rate and/or vorticity, the strength of the signal is dependent on the size of the prey, being proportional to the length of the prey or the length squared, respectively (Eqs. 8, 11 \& 15). Thus, larger prey would be able to detect a predator from a longer distance than a small one. Consistent with this, Landry (1978) found that the escape probability of copepods (various species) to a siphon suction flow increased with their length, irrespective of species. This, of course, could be due both to longer reaction distance, as predicted, and to more efficient escape reactions of larger copepods. Kiørboe et al. (1999) provided direct experimental evidence of the predicted size-effect for developmental stages of Acartia tonsa responding to fluid deformation rate.
There may be different threshold sensitivities between species that are unrelated to size (cf. Fields \& Yen 1997, Viitasalo et al. 1998) but the above suggests that there is a significant size effect. This does not necessarily imply that larger copepods are less susceptible to predation because larger copepods themselves generate stronger hydrodynamical signals and may, thus, be perceived by their predators at a longer distance.

\section{Prey perceiving predator: copepods detecting fish larvae}

Most larval fish are almost exclusively planktivorous and feed mainly on small copepods and copepod nauplii. We may think of the front part of a small fish as a translating (half)sphere. Thus, Fig. 3a-d may be a sufficiently good approximation of the fluid disturbances in front of a swimming larval fish. Upon prey location, which is visual, fish larvae typically approach their prey front on. Immediately in front of the approaching fish $(\theta=0)$ vorticity is zero, and in this idealised situation the fluid disturbance most likely to be perceived by small (mm-sized) prey is, therefore, deformation rate as given by Eq. (3)

In this situation, the reaction distance increases with the approach velocity of the predator, declines with increasing magnitude of the threshold deformation rate and is directly proportional to the size of the predator (C) (Eq. 18, Fig. 5b). The experiment of Wong (1996) provides a simple illustration of this latter prediction. The escape reaction distance of 2 species of freshwater copepods to a moving sphere varied in direct proportion to the size of the sphere.

Eqs. (3) \& (18) (Figs. 4 \& 5) may help us understand some characteristics of the predatory behaviour of larval fish. Evident in Fig. $4 \mathrm{~b}$ is a maximum deformation rate in front of a moving sphere, which is given by $3 U / 8 \mathrm{c}$. Consequently, there is a critical predator swimming velocity $\left(U_{C R}\right)$ below which a prey with a given threshold sensitivity $\left(\Delta^{*}\right)$ will be unable to sense the approaching predator, independent of how close it gets, $U_{C R}=8 c \Delta^{*} / 3$. Many larval fish are cruise predators, i.e. they swim at a more or less constant velocity during search for food particles. Because swimming velocities of larval fish are of the order of 1 body length $\mathrm{s}^{-1}$ (Miller et al. 1988), and since the ratio of head radius to body length is of the order of 0.05 to 0.1 , typical cruising velocities ( $U>10$ to $20 \mathrm{C} \mathrm{s}^{-1}$ ) exceed the critical velocity of prey with threshold sensitivities, $\Delta^{*}$, less than 5 to $10 \mathrm{~s}^{-1}$. Since most copepods appear to have threshold sensitivities less than that $\left(0.5\right.$ to $5.0 \mathrm{~s}^{-1}$, reviewed in Kiørboe et al. 1999), such a strategy would lead to escape reactions of the prey in front of the approaching predator and a very limited chance of 
prey capture. Zooplankters would react to an approaching predator swimming at velocity $U=15 \mathrm{C} \mathrm{s}^{-1}$ at distances between $1.0 \mathrm{C}\left(\Delta^{*}=5 \mathrm{~s}^{-1}\right)$ and $3.8 \mathrm{C}\left(\Delta^{*}=\right.$ $0.5 \mathrm{~s}^{-1}$ ) (Eq. 18). However, larval fish predators may circumvent this problem by being able to locate (visually) potential prey at even longer distances, and by reducing cruising speed when approaching prey location; both appear to be the case in larval and juvenile fish. The prey location distance in larval fish is generally of the order of 0.5 to 1.0 body length (Miller et al. 1988), or 5 to $10 \mathrm{c}$, well in excess of the reaction distance of the prey. After the prey has been located, the larval fish typically form an attack posture and very slowly approaches the prey until arriving at strike distance (Munk \& Kiørboe 1985, Munk 1992). The lowered approach velocity reduces the distance at which the prey reacts to the approaching predator, and may allow the predator to come unnoticed up to the strike distance. Viitasalo et al. (1998) described the similar but slightly different behaviour of small $(c=0.1 \mathrm{~cm}) 3$ spined stickleback preying upon copepods (Eurytemora). Upon prey location $(>2 \mathrm{~cm})$, the fish approaches the prey front on while slowing down, such that the final approach speed is reduced by a factor of about 5 to $\approx 1 \mathrm{~cm} \mathrm{~s}^{-1}$. The final approach speed is crucial for the predation attempt. The average final approach speed of unsuccessful attempts was $1.3 \mathrm{~cm}$ $\mathrm{s}^{-1}$ and the copepods reacted by escape at an average distance of (measured from tip of snout, i.e. $R-C$ ) $0.24 \mathrm{~cm}$ and before the fish came at strike distance. This is consistent with a threshold deformation rate of $1.5 \mathrm{~s}^{-1}$ (Eq. 3), which is close to that estimated in independent experiments $\left(2 \mathrm{~s}^{-1}\right)$. The observed threshold deformation rate further predicts that the fish should approach with a velocity less than $0.53 \mathrm{~cm} \mathrm{~s}^{-1}$ to remain undetected up to the strike distance $(0.08 \mathrm{~cm})$. This is close to the average final approach speed of successful attacks, $0.48 \mathrm{~cm} \mathrm{~s}^{-1}$. Another copepod (Temora), with a higher estimated threshold deformation rate $\left(8 \mathrm{~s}^{-1}\right)$, was unable to perceive the approaching fish and, thus, never managed to escape. This is also consistent with our model.

\section{Predator perceiving prey: ambush feeding copepods}

Since chemosensory perception of small prey particles requires a feeding current (Andrews 1983), ambush and cruise predators most likely perceive their prey by the hydromechanical disturbance they generate. The simplest situation, to be considered below, is a stationary ambush predator, such as Oithona, that feeds on sinking or swimming particles.

We assume that the predator reacts to the absolute magnitude of the fluid velocity at some velocity thresh- old, $u^{*}$, and Eqs. (24) \& (25) yield expressions for reaction distance (cf. Fig. 5). Several generalizations can be derived. First, prey particles with velocities $U<u^{*}$ will never be perceived. Thus, there is a lower threshold of the prey velocity - and, hence, size-spectrum - which is determined simply by the prey sinking/swimming velocity

Secondly, prey velocity is much more important for prey encounter rates than traditionally anticipated. Because good approximations (for $R \gg c$ ) of the reaction distances (Eqs, $24 \& 25$ ) are $-3 c U / 2 u^{*}$ and $3 c U / 4 u^{*}$ for front and sidewards prey detection, respectively, it follows that the reaction distance is approximately proportional to $U$. For an ambush predator, the clearance rate $(\beta)$ scales with the reaction distance squared (depending on the geometry of the perception volume, though) and with the prey velocity, $\beta \propto R^{2} U$. However, because $R$ scales with $U$, clearance scales with $U^{3}$. Likewise for a cruise predator with swimming velocity $V, \beta \propto R^{2}\left(U^{2}+V^{2}\right)^{0.5} \propto U^{2}\left(U^{2}+V^{2}\right)^{0.5}$. Models of prey encounter rates often ignore the effect of prey swimming velocity because $V \gg U$. However, since prey detection distance depends on prey velocity, this is not generally warranted.

Thirdly, encounter rates with sinking particles are strongly size dependent. From Eqs. (24) \& (25) it follows that the reaction distance is directly proportional to prey particle size, $c$. The sinking or swimming velocity of particles typically increases with particle size. For example, according to Stokes' law, particle sinking velocity scale with particle size squared. Therefore, since $U \propto c^{2}$ and $R \propto c U \propto c^{3}$, clearance rates of sinking particles, $\beta \propto R^{2} U \propto c^{8}$, scale with particle size raised to a power of 8 ! Thus, stationary flux feeders perceiving sinking particles by means of the fluid disturbances generated by the prey should be very much biased towards large particles. The scaling factor may be somewhat relaxed, depending on the exact geometry of the perceptive volume, and because the density of biological particles typically declines somewhat with size. Also, there is a maximum size of particles that a copepod can handle and consume. Yet, these considerations suggest a substantial size effect. Flux feeding copepods, such as Oithona (see below) or Neocalanus cristatus (Dagg 1993), may therefore provide quite efficient 'filters' for large sinking marine snow particles and fecal pellets.

As an example of a copepod ambush predator we will examine the case for Oithona similis. This copepod does not produce a feeding current (Paffenhöfer 1993) but hangs quietly and motionless in the water while slowly sinking. Videorecordings have demonstrated that it can perceive particles without direct contact, both by the antennules and by the telson (Svensen \& Halvorsen unpubl.). For simplicity its sinking velocity 
is, in the following, assumed to be zero. We further assume that $O$. similis perceives prey that are within one reaction distance of the antennae or the telson. Therefore, clearance rate $\beta=2 R d U$, where $d$ is the sum of the telson length and the span of the antennae $(-1.5 \mathrm{~mm})$.

Feeding on motile prey. Oithona similis feeds on a variety of protists, but apparently mainly on motile species (Sabatini \& Kiørboe 1995). Nakamura \& Turner (1997) measured clearance rates of $O$. similis fed various motile flagellates and ciliates. They found that 2 to $8 \mu \mathrm{m}$ sized flagellates were not cleared significantly, and that the clearance of larger prey increased with prey size (Fig. 6). A lower threshold of the size spectrum is consistent with our model, since swimming velocity increases with size in protists (Hansen et al. 1997) and because prey with swimming velocities $<u^{*}$ will not be perceived.

Nakamura \& Turner (1997) found that the smallest prey that were cleared significantly had a cell volume of $4.2 \times 10^{2} \mu^{3}(c \approx 4.6 \mu \mathrm{m})$. Hansen et al. (1997) compiled swimming velocities of flagellates and ciliates and applying their generalized size-swimming velocity equation implies that $u^{*}<0.013 \mathrm{~cm} \mathrm{~s}^{-1}$. Clearance rates predicted by assuming $u^{*}=0.004 \mathrm{~cm} \mathrm{~s}^{-1}$ and prey swimming velocities according to Hansen et al. (1997) correspond with those observed (Fig. 6), and the model quite accurately predicts the observed size dependency of clearance rates.

The model suggests that Oithona similis should be incapable of feeding on non-motile phytoplankters, except on very large species that sink at velocities exceeding $u^{*}$. If we assume phytoplankton sinking velocity to depend on cell size as $U=2.5 c^{117}$ (Jackson 1990), then the minimum size of immobile phytoplankters that can be perceived is $\left(u^{\bullet} / 2.5\right)^{0.85}=0.0042 \mathrm{~cm}$ for

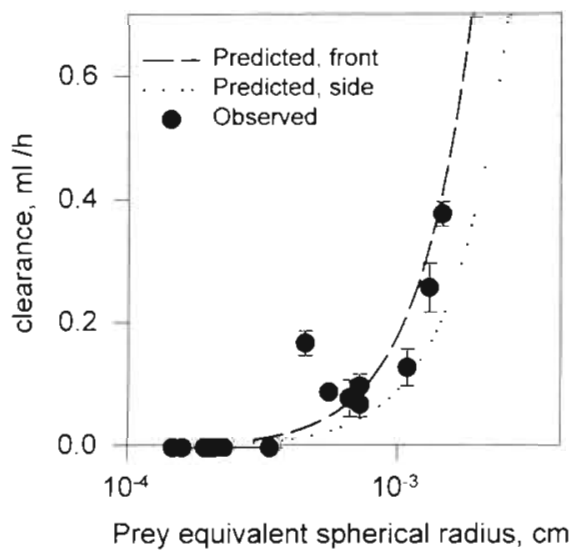

Fig. 6. Oithona similis clearance rates of different flagellates and ciliates as a function of their size. Data from Nakamura \& Turner (1997). The dotted lines show predicted clearance rates assuming $u^{*}=0.004 \mathrm{~cm} \mathrm{~s}^{-1}$ and that the prey is perceived from its front and its side, respectively. Logiswimming speed, $\left.\mu \mathrm{m} \mathrm{s}^{-1}\right)=1.61+0.195 \log \left(\right.$ cell volume, $\left.\mu \mathrm{m}^{3}\right)$ $u^{*}=0.004 \mathrm{~cm} \mathrm{~s}^{-1}$. Thus, according to the model, $O$ similis can only feed on immobile phytoplankters with a diameter exceeding about $80 \mu \mathrm{m}$, consistent with the apparent lack of feeding on small immobile diatoms.

Flux feeding. Oithona similis feeds intensively on copepod faecal pellets and Gonzáles \& Smetacek (1994) suggested that $O$. similis could be quantitatively important in retarding the vertical flux of sinking pellets and, hence, cause retention of organic material in the euphotic zone that would otherwise be 'Iost'. In the following we examine $O$. similis flux feeding be means of the model

The density of calanoid fecal pellets is about $1.15 \mathrm{~g}$ $\mathrm{cm}^{-3}$ (Butler \& Dam 1994). Assuming (for simplicity) spherical shape, the settling velocity $\left(\mathrm{cm} \mathrm{s}^{-1}\right)$ is therefore $4300 c^{2}$ (Stokes' law). Combining this expression of settling velocity with the equations for reaction distance (Eqs. $24 \& 25)$ and clearance $(\beta=2 R d U$ ), and assuming $u^{*}=0.004 \mathrm{~cm} \mathrm{~s}^{-1}$ as estimated above, yields a predicted relation between clearance rate and fecal pellet size (Fig. 7). Pellets with sinking velocities less than $u^{*}$ will not be perceived, and the critical pellet size for detection, $c_{C R}=\left(u^{*} / 4300\right)^{0.5}=0.00096 \mathrm{~cm}=$ $9.6 \mu \mathrm{m}$. Above that size predicted clearance rate on pellets increases dramatically with size, from about $15 \mathrm{ml} \mathrm{d}^{-1}$ for an Acartia sized pellet $(c=0.0025 \mathrm{~cm}$ ) to about $1000 \mathrm{ml} \mathrm{d}^{-1}$ for a Calanus sized pellet $(c=$ $0.006 \mathrm{~cm}$ ) (Fig. 7), The magnitude and size-dependency of the scarce observations of fecal pellet clearance rates accord well with this prediction, both for experimentally determined clearance rates (based on data in Gonzáles \& Smetacek (1994) and from Svensen \& Halvorsen unpubl.) and for those that can be derived from field observations (based on data in Gonzáles \& Smetacek 1994) (Fig. 7). Such high clearance rates of copepod fecal pellets would imply that typical densities of Oithona similis in temperate and subarctic oceans $\left(10^{4}\right.$ to $10^{6} \mathrm{~m}^{-2}$ (Hay et al. 1991, Hopkins et al 1993, Gonzáles \& Smetacek 1994, Kiørboe \& Nielsen 1994), may efficiently block the flux of - in particular-large fecal pellets in the ocean. Given the almost ubiquitous presence of Oithona sp. in the ocean, this prediction is consistent with the frequent observation that only a small fraction of copepod fecal pellets reach the sea floor (e.g. Smetacek 1980, Bathmann et al 1987. Gonzáles et al. 1994). Also, contrary to intuition, the testable prediction is that even though large pellets flux faster than smaller ones, the material contained in large pellets is retained within the euphotic zone with higher efficiency than that contained in smaller pellets due to this mechanism. This is different from the effect of passive flux feeders, such as pteropods (cf. Jackson 1993), that retain particles of different sizes with almost equal efficiency. These important processes obviously need further study. 


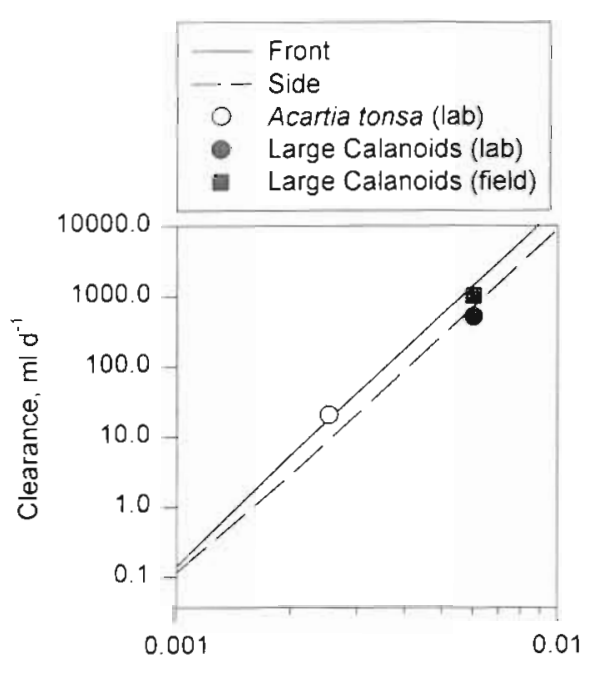

Fecal pellet equivalent spherical radius, $\mathrm{cm}$
Fig. 7 Oithona similis. Predicted and observed clearance rates of copepod faecal pellets as a function of their size. The prediction assumes that $v^{*}=$ $0.004 \mathrm{~cm} \mathrm{~s}^{-1}, U=4300 c^{2}$ ( $C=$ faecal pellet equivalent spherical radius), and that the sinking faecal pellet is perceived from its front and its side, respectively. The 2 laboratory observations of unsaturated clearance rates of calanoid faecal pellets are based on Gonzáles \& Smetacek (1994) and Svensen \& Halvorsen (unpubl.). These estimates were derived from observed concentrations $\left(C_{t}\right)$ of calanoid faecal pellet after $24 \mathrm{~h}$ incubations of calanoid copepods (large calanoids and Acartia tonsa, respectively) with and without $O$. similis females, by assuming that calanoid faecal pellet production rate ( $f$, per unit volume and time) was constant over time and that pellets remained suspended in the incubation chambers; $C_{l}=f / \alpha\left(1-\mathrm{e}^{-\alpha t}\right)$, where $\alpha$ is the fractional clearance rate due to all Oithona present in the incubation bottle and $t$ is the incubation time. The field estimate of clearance rate is based on station-averaged observations of calanoid faecal pellet production rate $(A)$, faecal pellet concentration $(B)$, fractional pellet flux rate $(C)$, and concentration of $O$. similis $(D)$; clearance $=[A / B-C / / D$. These data were from chlorophyll-rich stations in the Barents Sea (Gonzáles \& Smetacek 1994)
Thus, this simple model can accommodate the 3 orders of magnitude variation in clearance rate observed in Oithona similis feeding on both motile prey and sinking fecal pellets ( 1 to $1000 \mathrm{ml} \mathrm{d}^{-1}$ ), it explains essential features of Oithona feeding biology, and it has implications to our understanding of vertical particle fluxes in the ocean.

\section{Summary and conclusions}

This work has proposed that prey and predator perception in small planktonic copepods depend on different characteristics of the fluid disturbance generated by prey and predator, respectively. While prey perception rely on the magnitude of the fluid velocity, predator perception depends on one or several characteristics related to velocity gradients (maximum deformation rate, vorticity and/or acceleration). All these components cause quantifiable signals to the copepod in the form of velocity differences.

We have demonstrated that prey perception and, hence, prey encounter rates are strongly biased towards larger prey particles. We have considered copepod feeding on sinking faecal pellets, but similar considerations may apply to how the numerous zooplankters (including copepods) that colonise marine snow particles actually locate these. Colonisation and remineralisation rates of large sinking particles are quantitatively important (Kiørboe 1998) and have obvious implications for the vertical particle flux in the ocean, but the mechanisms involved are poorly understood. The results of this study may provide a starting point for further exploration of this important issue. We have also demonstrated that velocities of moving prey not only influence prey encounter rates directly, but also indirectly, by increasing prey perception distances. This latter effect may be the more important one, but it has largely been ignored in previous work (see Tiselius et al. 1997, however).

Hydromechanical perception of predators depend on the intensity of velocity gradients and, therefore, also on the size of the prey. The implication of this sizedependency requires future attention. While we have identified the components of velocity gradients that may be perceived by a copepod and quantified signal strengths, it is unclear from the present (and earlier) work which component of the velocity gradient provides the signal that actually triggers the escape response. Fluid shear is often assumed to constitute the triggering signal (cf. Fields \& Yen 1996, 1997) but it is only relevant in very special situations and should not be applied generally. Signal strengths due to vorticity and deformation rate are much larger than those due to acceleration in small copepods. Undisturbed prey swimming and sinking behaviour cause velocity differences between the copepod and the ambient fluid which may be difficult or impossible to distinguish from the signals related to vorticity and acceleration. Ockham's razor would therefore suggest that deformation rate is generally the most significant component. However, experiments and observations are required to elucidate this. In a subsequent paper we report on such observations (Kiørboe et al. 1999).

Acknowledgements. We are much indebted to P. Jonsson, Enric Saiz, David Fields, and Josefine Titelman for discussions and comments to the manuscript. We received financial support from the Danish Natural Science Research Council (\# 9502163) and from the EU-MAST project KEYCOP (MAS3CT97-0148) 
Appendix 1. Relative motion near a point

Deformation and rotation are aspects of the relative motion of fluid elements in a small region of space. Specifically, the relative velocity $\delta \mathbf{u}$, of 2 fluid elements can be written as a linear function of their separation $\delta \mathrm{r}$ :

$$
\delta \mathbf{u}=\mathbf{e} \cdot \delta \mathbf{r}+\omega \times \delta \mathbf{r}
$$

(e.g. Batchelor 1967, Acheson 1990). The $3 \times 3$ matrix e, represents the rate of strain of the fluid flow (e.g. Pedley 1997). This is also called the rate of deformation. The diagonal components of this matrix represent the deformation rate in the co-ordinate directions. This is most easily envisages as how a spherical volume is deformed into an ellipsoid. The off-diagonal components can be interpreted similarly, and represent the deformation rate in the transverse directions. A co-ordinate rotation can always be performed to realign these transverse components with some transformed coordinates. That is, the matrix e can be diagonalised. When in diagonal form, the elements along the diagonal are the principal components of the rate of strain.

The 3-vector $\omega$ in Eq. (A1) is the vorticity of the fluid and is defined in the usual way as $\omega=\nabla \times \mathbf{u}$. That feature of the flow field represented in the rate of strain tensor is solely responsible for the motion of fluid elements relative to each other. The vorticity contribution represents solid body rotation, and thus, no relative motion of fluid elements. Note that a solid body, rotating about the $x_{3}$ axis at a rate $\Omega$, has a vorticity component along $\omega_{3}$ along this axis equal to twice the rotation rate. That is $\omega_{3}=2 \Omega$

\section{Axial symmetric flow and spherical co-ordinates}

Much of the formulation within this work is facilitated with the use of spherical co-ordinates $(r, \theta, \phi)$, where $r$ is the distance from the origin, $\theta$ the polar angle, and $\phi$ is the azimuthal about the axis $\theta=0$. Each of the co-ordinated has an associated unit vector $\hat{\mathbf{r}}_{,}, \hat{\theta}, \hat{\phi}$. If we think of the earth, then these would be in the up, south and east directions respectively.

In spherical co-ordinates, vector operators have a somewhat different form than they do in the more commonly used Cartesian co-ordinates. Within this work we consider only flow fields with no component and no variation in the $\phi$ coordinate, i.e. a flow that is axially symmetric about the axis $\theta$ $=0$. In this case, we can write the velocity vector $\mathbf{u}$ as

$$
\mathbf{u}(r, \theta)=\hat{\mathbf{r}} u_{r}+\hat{\theta} u_{\theta}
$$

The general expressions for the components of the rate of strain matrix, i.e.

$$
\mathrm{e}=\left(\begin{array}{lll}
e_{r r} & e_{r \theta} & e_{r \phi} \\
e_{r \theta} & e_{\theta \theta} & e_{\theta \phi} \\
e_{r \phi} & e_{\theta \phi} & e_{\oplus \phi}
\end{array}\right)
$$

are then given by:

$$
\begin{array}{ll}
e_{r r}=\frac{\partial u_{r}}{\partial r} \quad e_{\theta \theta}=\frac{1}{r} \frac{\partial u_{\theta}}{\partial \theta}+\frac{u_{t}}{r} & e_{\phi}=\frac{u_{r}}{r}+\frac{u_{\theta}}{r} \cot \theta \\
e_{r \theta}=\frac{r}{2} \frac{\partial}{\partial r}\left(\frac{u_{\theta}}{r}\right)+\frac{1}{2 r} \frac{\partial u_{r}}{\partial \theta} & e_{r \theta}=e_{\theta \oplus}=0
\end{array}
$$

(e.g. Batchelor 1967, p. 601, Acheson 1990, p. 355). The principal components of the rate of strain, $\delta_{n}(n=1,2,3)$, are given by

$$
\operatorname{det}\left(\begin{array}{ccc}
e_{r r}-\delta_{n} & e_{r \theta} & 0 \\
e_{r \theta} & e_{\theta \theta}-\delta_{n} & 0 \\
0 & 0 & e_{\phi \theta}-\delta_{n}
\end{array}\right)=0
$$

where det represents the determinant of the matrix (e.g. Hohn 1973). That is,

$$
\begin{aligned}
& \delta_{1,2}=\frac{1}{2}\left\{\left(e_{r r}+e_{\theta \theta}\right) \pm \sqrt{\left(e_{r r}-e_{\theta \theta}\right)^{2}+4 e_{r \theta}^{2}}\right\} \\
& \delta_{3}=e_{\phi \theta}
\end{aligned}
$$

In this work, we define the maximum deformation rate as

$$
\Delta=\operatorname{MAX}\left(\left|\delta_{1}\right|,\left|\delta_{2}\right|,\left|\delta_{3}\right|\right)
$$

i.e. the maximum of the absolute value of the 3 principal components of the rate of strain.

Other general expressions of interest for axially symmetric flow can be deduced from equations given in Batchelor 1967, p. 601 . Specifically, we can write vorticity

$$
\omega=\nabla \times \mathbf{u}=\hat{\phi} \omega_{\phi}=\hat{\phi} \frac{1}{r}\left\{\frac{\partial\left(r u_{\theta}\right)}{\partial r}-\frac{\partial u_{r}}{\partial \theta}\right\}
$$

and advective acceleration

$$
\begin{aligned}
& \mathbf{a}=\mathbf{u} \cdot \nabla \mathbf{u} \\
& =\hat{\mathbf{r}}\left\{u_{r} \frac{\partial u_{r}}{\partial r}+\frac{u_{\theta}}{r} \frac{\partial u_{r}}{\partial \theta}-\frac{u_{\theta}{ }^{2}}{r}\right\}+\hat{\theta}\left\{u_{r} \frac{\partial u_{r}}{\partial r}+\frac{u_{\theta}}{r} \frac{\partial u_{\theta}}{\partial \theta}+\frac{u_{\theta} u_{r}}{r}\right\}
\end{aligned}
$$

\section{Creeping flow around a sphere}

A.t low Reynolds number (i.e. negligible inertia), the flow of an incompressible, viscous fluid around a stationary sphere of radius $c$ is given by the stream function

$$
\psi(r, \theta)=-\frac{1}{2} U r^{2} \sin ^{2} \theta\left\{1-\frac{3 c}{2 r}+\frac{c^{3}}{2 r^{3}}\right\}
$$

(e.g. Acheson 1990, p. 225) where $U$ is the flow velocity well away from the sphere. These streamlines are depicted in Fig. $2 \mathrm{a}$. The velocity components are given as

$$
\begin{gathered}
u_{r}=\mathbf{u} \cdot \hat{\mathbf{r}}=\frac{1}{r^{2} \sin \theta} \frac{\partial \psi}{\partial \theta}=-U \cos \theta\left\{1-\frac{3 c}{2 r}+\frac{c^{3}}{2 r^{3}}\right\} \\
u_{\theta}=\mathbf{u} \cdot \hat{\theta}=\frac{-1}{r \sin \theta} \frac{\partial \psi}{\partial \theta}=U \sin \theta\left\{1-\frac{3 c}{4 r}-\frac{c^{3}}{4 r^{3}}\right\}
\end{gathered}
$$

For this case, from Eq. (A9), vorticity is given as

$$
\omega_{\phi}=U \sin \theta \frac{3 c}{2 r^{2}}
$$

and, from Eq. (A4), the rate of strain matrix as

$$
\mathrm{e}=U \frac{3 c}{4 r^{4}}\left(\begin{array}{ccc}
-2\left(r^{2}-c^{2}\right) \cos \theta & c^{2} \sin \theta & 0 \\
c^{2} \sin \theta & \left(r^{2}-c^{2}\right) \cos \theta & 0 \\
0 & 0 & \left(r^{2}-c^{2}\right) \cos \theta
\end{array}\right)
$$

We note that directly in front of the sphere $(\theta=0)$, the maximum deformation rate $\Delta$ is $\left|e_{r s}\right|$, corresponding to the top left element in the matrix in Eq. (A15). Thus, from Eq. (A4), we have $\Delta(\theta=0)=\left|\delta u_{r} / \delta r\right|$

Substituting Eq. (A12) \& (A13) into Eq. (A10) and after some manipulation, the acceleration can be written as 
Appendix 1 (continued)

$$
\begin{aligned}
& \mathbf{u} \cdot \nabla \mathbf{u}= \\
& \dot{\mathbf{r}} U^{2} \frac{3}{16 r^{7}} c(r-c)^{2}(r+c)\left\{(4 r-c)(2 r+c) \cos ^{2} \theta-\left(c^{2}+c r+4 r^{2}\right) \sin ^{2} \theta\right\} \\
& +\dot{\theta} U^{2} \frac{3}{16 r^{7}} c^{2}(r-c)^{3}(3 r+c) \sin \theta \cos \theta
\end{aligned}
$$

\section{A translating sphere: feeding current analogy}

A physical problem related to the creeping flow around a sphere is that of a translating sphere at low Reynolds number. The solution to this problem is the stream function

$$
\psi(r, \theta)=-\frac{1}{2} U r^{2} \sin ^{2} \theta\left\{\frac{3 c}{2 r}-\frac{c^{3}}{2 r^{3}}\right\}
$$

which gives the velocity components

$$
\begin{aligned}
& u_{r}=\mathbf{u} \cdot \hat{\mathbf{r}}=\frac{1}{r^{2} \sin \theta} \frac{\partial \psi}{\partial \theta}=-U \cos \theta\left\{\frac{3 c}{2 r}-\frac{c^{3}}{2 r^{3}}\right\} \\
& u_{\theta}=\mathbf{u} \cdot \hat{\theta}=\frac{-1}{r \sin \theta} \frac{\partial \psi}{\partial r}=U \sin \theta\left\{\frac{3 c}{4 r}+\frac{c^{3}}{4 r^{3}}\right\}
\end{aligned}
$$

Formally, this means that the sphere is moving with a velocity $U$ in an otherwise still fluid. The same solution also describes the situation where the sphere is stationary and fluid flows through it at a velocity $-U$. This is analogous to a low Reynolds number feeding current.

It turns out that the difference between vorticity and rate of strain for this case, and that for the flow around the sphere, is a change of sign. The only significant difference appears in the acceleration term, which for this case becomes

$$
\begin{aligned}
& \mathbf{a}=\mathbf{u} \cdot \nabla \mathbf{u}= \\
& \hat{\mathbf{r}} U^{2} \frac{3}{16 r^{7}} c\left(r^{2}-c^{2}\right)\left\{-4\left(3 r^{2}-c^{2}\right) \cos ^{2} \theta+\left(3 r^{2}+c^{2}\right) \sin ^{2} \theta\right\} \\
& +\hat{\theta} U^{2} \frac{3}{16 r^{7}} c^{2}\left(3 r^{4}+6 c^{2} r^{2}-c^{4}\right) \sin \theta \cos \theta
\end{aligned}
$$

Specific evaluations are

$$
\begin{aligned}
& \text { at } \theta=0, \quad \mathbf{a}=\mathbf{u} \cdot \nabla \mathbf{u}=-\hat{\mathbf{r}} U^{2} \frac{3}{4 r^{7}} c\left(r^{2}-c^{2}\right)\left(3 r^{2}-c^{2}\right) \\
& \text { at } \theta=\pi / 2, \quad \mathbf{a}=\mathbf{u} \cdot \nabla \mathbf{u}=\hat{\mathbf{r}} U^{2} \frac{3}{16 r^{7}} c\left(r^{2}-c^{2}\right)\left(3 r^{2}+c^{2}\right)
\end{aligned}
$$

All formulae were checked using Mathematica ${ }^{(2)}$ v2.2 (1993) from Wolfram Research Inc.

\section{LITERATURE CITED}

Acheson DJ (1990) Elementary fluid dynamics. Clarendon Press, Oxford

Andrews JC (1983) Deformation of the active space in the low Reynolds number feeding current of calanoid copepods. Can J Fish Aquat Sci 40:1293-1302

Batchelor GK (1967) An introduction to fluid dynamics. Cambridge University Press, Cambridge

Bathmann UV, Noji TT Peinert R (1987) Copepod faecal pellets: abundance, sedimentation and content at a permanent station in the Norwegian Sea in May/June 1986. Mar Ecol Prog Ser 38:45-51

Butler M, Dam HG (1994) Production rates and characteristics of faecal pellets of the copepod Acartia tonsa under simulated phytoplankton bloom conditions: implications for vertical fluxes. Mar Ecol Prog Ser 114:81-89

Dagg M (1993) Sinking particles as a possible food source of nutrition for the large calanoid copepod Neocalanus cristatus in the subarctic Pacific Ocean. Deep-Sea Res 40: $1431-1445$

Fields DM, Yen J (1993) Outer limits and inner structure: the 3-dimensional flow field of Pleuromamma xiphias (Calanoida: Metridinidae). Bull Mar Sci 53:84-95

Fields DM, Yen J (1996) The escape behaviour of Pleuromamma xiphias in response to a quantifiable fluid mechanical disturbance. In: Lenz PH, Hartline DK, Purcell JE, Macmillan DL (eds) Zooplankton: sensory ecology and physiology, Vol 1. Gordon and Breach Publishers, Amsterdam, p 323-340

Fields DM, Yen J (1997) The escape behaviour of marine copepods in response to a quantifiable fluid mechanical disturbance. J. Plankton Res 19:1289-1304

Gonzáles HE, Smetacek V (1994) The possible role of the cyclopoid copepod Oithona in retarding vertical flux of zooplankton faecal material. Mar Ecol Prog Ser 113:233-246
Gonzáles HE, Gonzáles SR, Brummer GJA (1994) Short-term sedimentation pattern of zooplankton, faeces and microplankton at a permanent station in Bjørnafjord (Norway) during April-May 1992. Mar Ecol Prog Ser 105:31-45

Hansen PJ, Bjørnsen PK, Hansen BW (1997) Zooplankton grazing and growth: Scaling within the $2-2,000-\mu \mathrm{m}$ body size range. Limnol Oceanogr 42:687-704

Haury LR, Kenyon DE, Brooks (1980) Experimental evaluation of the avoidance reaction of Calanus finmarchicus. J Plankton Res 2:187-202

Hay SJ, Kiørboe T, Matthews A (1991) Zooplankton biomass and production in the North Sea during the autumn circulation experiments, October 1987-March 1988. Cont Shelf Res 11:1453-1476

Heuch PA, Karlsen HE (1997) Detection of infrasonic water oscillations by copepodids of Lepeoptherius salmonis (Copepoda: Caligida). J Plankton Res 19:735-747

Hohn FE (1973) Elementary matrix algebra. The Macmillan Company, New York

Hopkins TL, Lancraft TM, Torres JJ Donnelly J (1993) Community structure and trophic ecology of zooplankton in the Scotia Sea marginal ice zone in winter (1988). Deep-Sea Res I 40:81-105

Jackson GA (1990) A model of the formation of marine algal flocs by physical coagulation processes. Deep-Sea Res 37: $1197-1211$

Jackson GA (1993) Flux feeding as a possible mechanism for zooplankton grazing and its implication for vertical particle flux. Limnol Oceanogr 38:1328-1331

Jonsson PR, André C, Lindegarth M (1991) Swimming behaviour of marine bivalve larvae in a flume boundary-layer flow: evidence for near-bottom confinement. Mar Ecol Prog Ser 79:67-76

Kiørboe T (1998) Population regulation and role of mesozooplankton in shaping marine pelagic food webs. Hydrobiologia 363:13-27 
Kiørboe T, Nielsen TG (1994) Regulation of zooplankton biomass and production in a temperate, coastal ecosystem. 1 Copepods. Limnol Oceanogr 39:493-507

Kiorboe T, Titelman J (1998) Feeding, prey selection and prey encounter mechanisms in the heterotrophic dinoflagellate Noctiluca scintillans. J Plankton Res 20:1615-1636

Kiørboe T, Saiz E, Visser A (1999) Hydrodynamic signal perception in the copepod Acartia tonsa. Mar Ecol Prog Ser 179:97-111

Kirk KL (1985) Water flows produced by Daphnia and Diaptomus: implications for prey selection by mechanosensory predators. Limnol Oceanogr 30679-686

Lamb H (1932) Hydrodynamics. Dover Publications, New York

Landry MR (1978) Predatory feeding behaviour of a marine copepod, Labidocera trispinosa. Limnol Oceanogr 23: $1103-1113$

Maxey MR, Riley JJ (1983) Equations of motion for a small rigid sphere in a nonuniform flow. Phys Fluids 26 883-889

Miller TJ, Crowder LB, Rice JA, Marshall EA (1988) Larval size and recruitment mechanisms in fishes: towards a conceptual framework. Can J Fish Aquat Sci 45:1657-1670

Munk P (1992) Foraging behaviour and prey size spectra of larval herring Clupea harengus. Mar Ecol Prog Ser 80: 149-158

Munk P, Kiørboe T (1985) Feeding behaviour and swimming activity of larval herring (Clupea harengus) in relation to density of copepod nauplii. Mar Ecol Prog Ser 24:15-21

Nakamura Y, Turner JT (1997) Predation and respiration by the small cyclopoid copepod Oithona similis: how important is feeding on ciliates and heterotrophic flagellates? J Plankton Res 19:1275-1288

Paffenhöfer GA (1993) On the ecology of marine cyclopoid copepods (Crustacea, Copepoda, Cyclopoida). J Plankton Res 15:37-55

Pedley TJ (1997) Introduction to fluid dynamics. Sci Mar 61(Suppl 1):7-24

Ploug H, Jørgensen BB (1999) A net-jet flow system for mass transfer and microsensor studies of sinking aggregates. Mar Ecol Prog Ser 176:279-290

Editorial responsibility: Otto Kinne (Editor).

Oldendorf/Luhe, Germany
Sabatini M, Kiørboe T (1994) Egg production, growth and development of the cyclopoid copepod Oithona similis. J Plankton Res 16:1329-1351

Schröder VR (1967) Verhalten von Cyclops abyssorum in der Strömung. Arch Hydrobiol Suppl 33:84-91

Smetacek V (1980) Zooplankton standing stock, copepod faecal pellets and particulate detritus in Kiel Bight. Estuar Coast Mar Sci 11:477-490

Strickler JR, Bal AK (1973) Setae of the first antennae of the copepod Cyclops scutifer (Sars): their structure and importance. Proc Natl Acad Sci USA 70:2656-2659

Tiselius P, Jonsson P (1990) Foraging behaviour of six calanoid copepods: observations and hydrodynamic analysis. Mar Ecol Prog Ser 66:23-33

Tiselius P, Jonsson P, Kaartved S, Olsen EM, Jørstad T (1997) Effects of copepod foraging behaviour on predation risk: an experimental study of the predatory copepod Pareuchaete norvegica feeding on Acartia clausi and $A$. tonsa (Copepoda). Limnol Oceanogr 42:164-170

Van Dyke M (1988) An album of fluid motion. The Parabolic Press, Stanford

Viitasalo M, Kiørboe T, Flinkman J, Pedersen LW, Visser AW (1998) Predation vulnerability of planktonic copepods: consequences of predator foraging strategies and prey sensory abilities. Mar Ecol Prog Ser 175:129-142

Wong CK (1996) Response of copepods to hydromechanical stimuli. Crustaceana 69:853-859

Yen J, Fields DM (1992) Escape response of Acartia hudsonica (Copepoda) nauplii from flow field of Temora longicornis (Copepoda). Arch Hydrobiol Beih Ergeb Limnol 36: $123-134$

Yen J, Strickler JR (1996) Advertisement and concealment in the plankton: what makes a copepod hydrodynamically conspicuous? Invertebr Biol 115:191-205

Yen J, Lenz, PH, Gassie DV, Hartline DK (1992) Mechanoreception in marine copepods: electrophysiological studies on the first antennae. J Plankton Res 14:459-512

Zaret RE (1980) Zooplankters and their interactions with water, with each other, and with their predators. PhD thesis, John Hopkins University, Baltimore, MD

Submitted: August 3, 1998; Accepted: November 18, 1998 Proofs received from author(s): March 25, 1999 